\title{
Grain size distribution at four developmental stages of crescent dunes in the hinterland of the Taklimakan Desert, China
}

\author{
JIA Wenru ${ }^{1}$, ZHANG Chunlai $^{1 *}$, LI Shengyu ${ }^{2}$, WANG Haifeng ${ }^{2}$, MA Xuexi ${ }^{2,3}$, WANG \\ Ningbo $^{2}$ \\ ${ }^{1}$ State Key Laboratory of Earth Surface Processes and Resource Ecology, Ministry of Education Engineering Center of \\ Desertification and Blown-sand Control, Beijing Normal University, Beijing 100875, China; \\ ${ }^{2}$ Xinjiang Institute of Ecology and Geography, Chinese Academy of Sciences, Urumqi 830011, China; \\ ${ }^{3}$ Xinjiang Academy of Forestry Science, Urumqi 830046, China
}

\begin{abstract}
Although scientists have performed many studies on crescent (barchan) dunes in the Taklimakan Desert, few studies reported the changes in grain size at different development stages of crescent dunes. In order to evaluate the changing trends of surface sediment grain size with dune development, we investigated the grain size characteristics at four developmental stages (oval sand pile, shield dune, incipient crescent dune and mature crescent dune) of crescent dunes by measuring the morphology of sand dune and observing the near-surface wind regime. The dunes have developed in a wide inter-dune corridor between high compound longitudinal ridges in China's Taklimakan Desert. The surface sediments at four developmental stages of the crescent dunes were primarily composed of fine sands, followed by very fine and medium sands. Mean grain sizes ranged from 2.8 to $3.1 \varphi$, with a unimodal distribution. The sands were moderately well-sorted, their distribution varied from platykurtic to very platykurtic, and symmetrical or skewed towards the fine particles. From oval sand piles through shield and incipient crescent dunes to mature crescent dunes, incipient grain size gradually increased, particles became finer, sorting became better, kurtosis and skewness increased. Grain sizes on the surface layer became coarser upwards from the toe of the windward slope and then became finer towards the bottom of the leeward slope. We found that the coarsest particles at different positions at the four developmental stages were different. The coarsest particles were distributed at the top of the oval sand piles and shield dunes, versus at the middle of the windward slope of the incipient and mature crescent dunes. Correlations between the mean grain size and other grain size parameters showed that as mean grain size became finer, sorting became better and kurtosis became wider, but skewness changed only slightly. In addition, grain size variation in the surface sediments correlated with the movement speed of the dunes in the study area. In the open ground among tall-complex longitudinal ridges in the hinterland of the Taklimakan Desert where aeolian environment is characterized by comparatively strong wind and unsaturated sand flow, faster dune movement corresponded to coarser grain size.
\end{abstract}

Keywords: crescent dune; developmental stage; grain size; Taklimakan Desert

Citation: JIA Wenru, ZHANG Chunlai, LI Shengyu, WANG Haifeng, MA Xuexi, WANG Ningbo. 2016. Grain size distribution at four developmental stages of crescent dunes in the hinterland of the Taklimakan Desert, China. Journal of Arid Land, 8(5): 722-733. doi: 10.1007/s40333-016-0016-9

\footnotetext{
"Corresponding author: ZHANG Chunlai (E-mail: clzhang@bnu.edu.cn)

Received 2015-12-26; revised 2016-04-16; accepted 2016-04-20

(C) Xinjiang Institute of Ecology and Geography, Chinese Academy of Sciences, Science Press and Springer-Verlag Berlin Heidelberg 2016
} 
Crescent sand dunes (also called barchan dunes) generally form where wind flows in a single direction and vegetation is sparse. Bagnold (1941) reported that under certain conditions, where the dune's cross-section is increasing in size, the initial rounded shapes of dunes is no longer stable, and the top and bottom of the dune's leeward slope moves faster than other parts, eventually leading to the formation of a slip face. Most researchers regard this as the classic model for development of crescent sand dunes. Wu (2003) and Ding (2010) described the developmental process of dunes as follows: from sand patches to oval sand piles, shield dunes, incipient crescent dunes and finally mature crescent dunes. We observed this same developmental sequence in the inter-dune corridors between high compound longitudinal ridges in the hinterland of the Taklimakan Desert.

High compound longitudinal sand ridges form the boundaries of wide inter-dune corridors in the hinterland of the Taklimakan Desert. In these 2 to $3 \mathrm{~km}$ wide inter-dune corridors, sand dunes are oriented from NNE to SSW. They are 200 to $500 \mathrm{~m}$ wide, 60 to $80 \mathrm{~m}$ tall and 5 to $6 \mathrm{~km}$ long. Moreover, in the inter-dune corridors, simple crescent dunes, barchan chains and longitudinal dunes are widely distributed (Zhu et al., 1981). Among these dune types, four main forms of simple crescent dunes are oval sand piles, shield, incipient crescent and mature crescent dunes, which represent the four stages of crescent dune development between sand patches and mature dunes (Wu, 2003; Ding, 2010).

The grain size distribution on dunes is not only related to its surface wind dynamics environment, but also associated with morphological characteristics of sand dunes (Lancaster, 1989; Livingstone, 1989; Pye and Tsoar, 1990; White and Tsoar, 1998; Lancaster et al., 2002; Nickling et al., 2002). Therefore, the grain size characteristics in the surface sediments are important in research on the morphological dynamics of dunes.

Crescent dune is a relatively simple dune form and has been studied in detail. Abundant data now exist on their formation, development, morphological characteristics, sand flow dynamics, and the characteristics of their sedimentary structure (Bagnold, 1941; Lancaster, 1985; Pye and Tsoar, 1990; Hesp and Hastings, 1998; Ling et al., 1998; Zhang et al., 1999; Ha et al., 2001). However, data on the variations in grain sizes in the surface sediments during different developmental stages were less reported. Accordingly, we aimed to explore the relationships between surface sediment characteristics and dune morphology during the formation and development of crescent dunes by analyzing the variation in grain sizes at different development stages, along the evolutionary spectrum from oval sand piles through shield and incipient crescent dunes, to mature crescent dunes.

\section{Study area and methods}

\subsection{Study area}

The Taklimakan Desert is the largest desert in China and the second largest shifting sand desert in the world. It covers an area of $33.78 \times 10^{4} \mathrm{~km}^{2}$, of which $82 \%$ is covered by various types of mobile dunes (Zhu et al., 1981). Our study area is located in the Tazhong mobile sand area in the desert's hinterland (38 $57^{\prime} 29^{\prime \prime}-38^{\circ} 59^{\prime} 47^{\prime \prime} \mathrm{N}, 83^{\circ} 42^{\prime} 54^{\prime \prime}-83^{\circ} 44^{\prime} 58^{\prime \prime} \mathrm{E}$; 1,127-1, $144 \mathrm{~m}$ asl; Fig. 1).

The dominant wind directions are from the ENE and NE throughout the year, and the primary sand-entraining winds occur in spring and summer. Using meteorological data from 1996 to 2000 provided by the Tazhong Meteorological Station which is located approximately $6.5 \mathrm{~km}$ from our study site, $\mathrm{Zu}$ et al. (2005) calculated the annual drift potential to be $114.5 \mathrm{VU}$, the resultant drift potential to be $67.0 \mathrm{VU}$ with a resultant drift direction to be $51.5^{\circ}$ in this area. Compound longitudinal dunes in the study area range from 30 to $70 \mathrm{~m}$ in height and in the trend of NE-SW direction, which is consistent with the dominant wind directions (Wang et al., 2003). The widest point in the inter-dune corridors was approximately 3,000 $\mathrm{m}$. Surface sediments are generally coarse, with a typical diameter of $0.5 \mathrm{~mm}$, but range up to fine gravels with a 2-mm diameter. Many simple crescent dunes are present in the inter-dune corridors. 


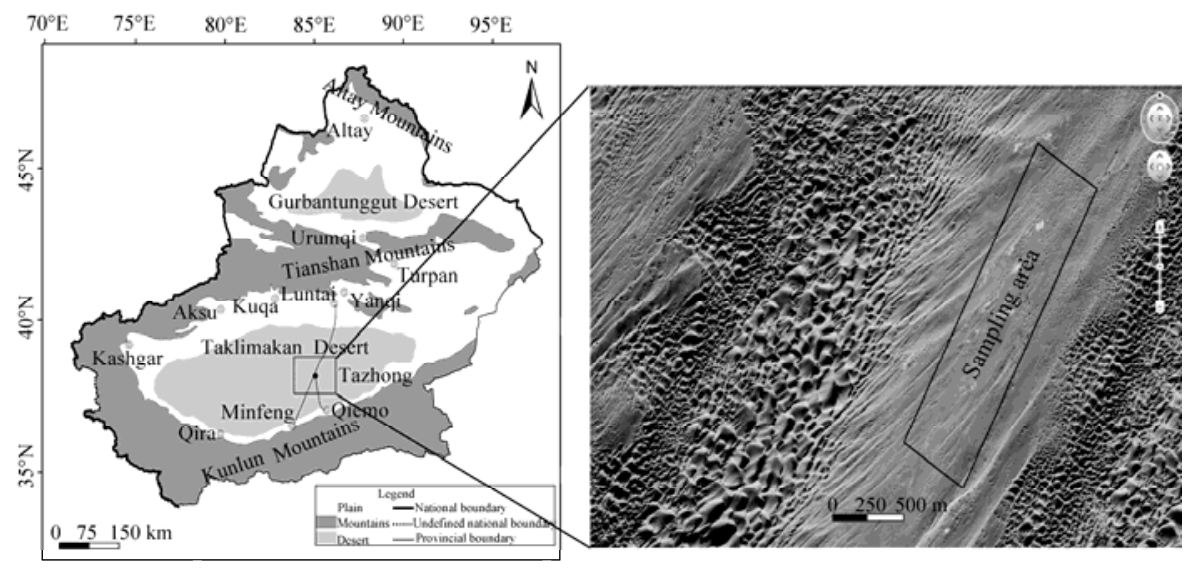

Fig. 1 Location of the study area and photo of the site

\subsection{Methods}

We selected 10 oval sand piles (OSP), 9 shield dunes (SD), 15 incipient crescent dunes (ICD) and 15 mature crescent dunes (MCD) along the ENE prevailing wind direction. We measured dune morphology using a GPS receiver with a precision of horizontal error $2.4 \mathrm{~mm} / \mathrm{km}$ and vertical error $1.2 \mathrm{~mm} / \mathrm{km}$ (X90, HUACE, Shanghai, China). Figure 2 illustrates the dune morphology, and Table 1 summarizes the morphological parameters of these classes of dune. To represent each developmental stage, we selected three dunes and constructed a grid using 90 -cm-long iron rods, which were inserted in the sediments to a depth of $50 \mathrm{~cm}$. The exposed height of the iron rods was measured six times per month to determine the depth of surface erosion or deposition. We used three-cup (2-cm in diameter) wind anemometers to observe wind velocities at a 2-m height at various dune locations. We installed a stepped sand sampler on each side of the anemometer to collect sand flow data at a collection height ranging from the ground surface to a height of $20 \mathrm{~cm}$; the sampler contained ten $2 \mathrm{~cm} \times 2 \mathrm{~cm}$ openings to collect blowing sand.

The distribution of sampling points on the dunes at each developmental stage was standardized in Fig. 2. P1 represents the toe of the windward slope, P2 the middle of the windward slope, P3 the top of the dune, P4 the middle of the leeward slope (or the top of the dune crest line for the incipient and mature crescent dunes), P5 the bottom of the leeward side (or the bottom of the slip face for the incipient and mature crescent dunes), P6 and P7 the edge of the left (ESE) and the right (WNW) sides of the dunes (Left and right are defined looking downwind from the windward side of the dune), P8 and P9 the tips of the left and right wings of the incipient and mature crescent dunes, and P10 the middle of the slip face of the mature crescent dunes.
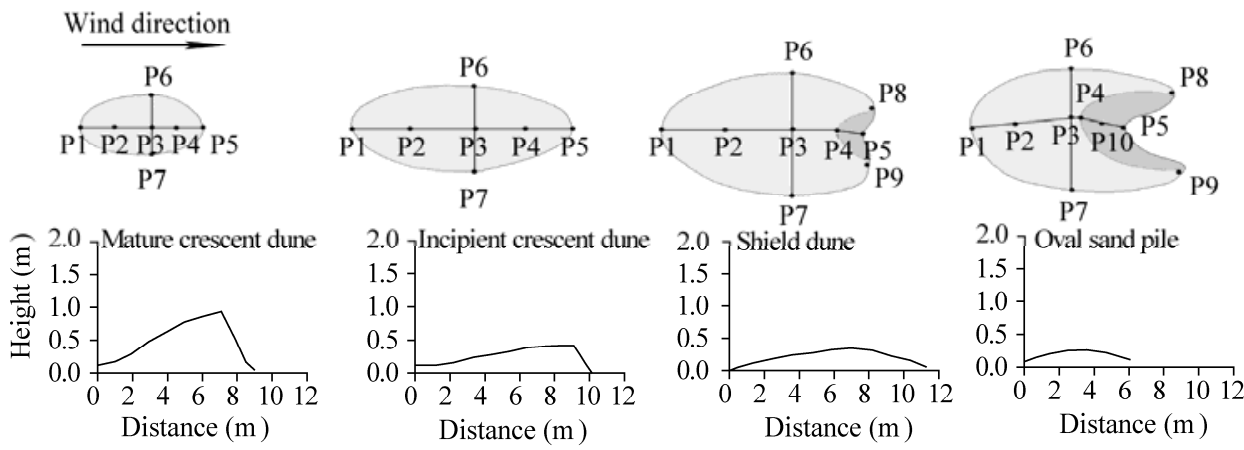

Fig. 2 Illustrations of typical dune profiles and locations of the sampling positions 
JIA Wenru et al.: Grain size distribution at four developmental stages of crescent dunes in the hinterland of...

Table 1 Morphological parameters of the four developmental stages of crescent dunes

\begin{tabular}{cccccccc}
\hline Development stage & Sample size & $\begin{array}{c}\text { Length of } \\
\text { windward slope }(\mathrm{m})\end{array}$ & $\begin{array}{c}\text { Length of } \\
\text { leeward slope }(\mathrm{m})\end{array}$ & $\begin{array}{c}\text { Left wing Right wing } \\
(\mathrm{m})\end{array}$ & $\begin{array}{c}\text { Width } \\
(\mathrm{m})\end{array}$ & $\begin{array}{c}\text { Height } \\
(\mathrm{m})\end{array}$ & $\begin{array}{c}\mathrm{m}) \\
\text { Oval sand pile }\end{array}$ \\
Shield dune & 10 & $3.4 \pm 0.6$ & $2.6 \pm 0.9$ & - & - & $3.9 \pm 0.3$ & $0.3 \pm 0.1$ \\
Incipient crescent dune & 15 & $7.1 \pm 2.2$ & $4.4 \pm 0.8$ & - & - & $5.6 \pm 0.6$ & $0.4 \pm 0.1$ \\
Mature crescent dune & 15 & $9.1 \pm 2.2$ & $1.0 \pm 0.4$ & $1.8 \pm 0.2$ & $1.6 \pm 0.3$ & $8.1 \pm 1.5$ & $0.4 \pm 0.1$ \\
\hline
\end{tabular}

Note: Mean \pm SD.

The sampling positions were separated into $20 \mathrm{~cm} \times 20 \mathrm{~cm}$ plots, and surface sediment was collected to a depth of $3 \mathrm{~cm}$ in each plot. In total, we collected 403 samples. Grain size analysis was conducted using a Mastersizer 2000 (Malvern Instruments Ltd., UK). The results of this particle analysis are presented as volume percentages. Based on the particle data, we calculated the mean grain size, sorting, skewness and kurtosis with $\varphi$ values according to the methods of Folk and Ward (1957).

\section{Results}

\subsection{Grain size composition}

The surface sediments in each of the four crescent dune developmental stages were mainly composed of fine sand (from 3 to $2 \varphi, 49 \%$ of the total), followed by very fine sand (4 to $3 \varphi$, $28 \%$ to $45 \%$ of the total). The medium sands accounted for no more than $18 \%$ of the total. Coarse silt and coarse sands accounted for less than $2 \%$ and less than $0.6 \%$ of the total, respectively. We found no particles finer than coarse silt or coarser than coarse sand. The proportions of very fine sands in the oval and shield dunes were $36.0 \%$ and $37.1 \%$, respectively, which were lower than those in incipient crescent dunes $(39.0 \%)$ and mature crescent dunes $(37.8 \%)$; in contrast, the proportions of medium sands in the oval and shield dunes $(9.1 \%$ in both) were higher than those in incipient crescent dunes $(6.2 \%)$ and mature crescent dunes $(7.1 \%)$. The other particle size classes did not account for a significant proportion of the total.

We found clear differences in the dominant grain sizes at the same positions on crescent dunes at different developmental stages (Table 2). The proportion of medium sand particles in the oval and shield dunes increased from the toe of the windward slope to the top of the dune and then decreased from the top of the dune to the bottom of the leeward slope. The tops of the oval and shield dunes contained a greater proportion of medium sand particles than the other positions (14.8\% and $17.7 \%$, respectively). In contrast, the proportion of medium sands in the incipient and mature crescent dunes increased from the toe of the windward slope towards the middle of the slope, then decreased again at the top of the dune. The toe of the windward slope and the bottom of the leeward slope of incipient crescent dunes had fewer medium sands than those at other positions. The top of the dune's crest line and the bottom of the leeward slope in mature crescent dunes contained a greater proportion of medium sands than those at other positions along the downwind axis of the dune.

The proportion of very fine sands first decreased and then increased along longitudinal axis of the dunes. At different stages of development, the position with the highest proportion of very fine sands changed. For example, oval and shield dunes had the most very fine sand at the bottom of the leeward slope, incipient crescent dunes had the most very fine sand at the toe of the windward slope, and mature crescent dunes had the most very fine sand at the top of the dune. The proportion of very fine sands on the left wing was generally higher than that on the right wing, but the pattern was reversed for the mature crescent dunes; however, the difference was small in each case. We found no clear trend in the proportion of fine sands at the four developmental stages. 
Table 2 Grain size distribution at different positions on the dunes (see Fig. 2 for details) at the four stages of crescent dune development

\begin{tabular}{|c|c|c|c|c|c|c|}
\hline \multirow[b]{2}{*}{ Development stage } & \multirow{2}{*}{$\begin{array}{l}\text { Sample } \\
\text { position }\end{array}$} & \multicolumn{5}{|c|}{ Proportion of total volume $(\%)$} \\
\hline & & $\begin{array}{c}\text { Coarse silt } \\
(5-4 \varphi)\end{array}$ & $\begin{array}{l}\text { Very fine sand } \\
\quad(4-3 \varphi)\end{array}$ & $\begin{array}{c}\text { Fine sand } \\
(3-2 \varphi)\end{array}$ & $\begin{array}{c}\text { Medium sand } \\
(2-1 \varphi)\end{array}$ & $\begin{array}{c}\text { Coarse sand } \\
(1-0 \varphi)\end{array}$ \\
\hline \multirow[t]{8}{*}{ Oval sand pile } & $\mathrm{P} 1$ & 1.09 & 39.12 & 54.19 & 5.60 & 0.00 \\
\hline & $\mathrm{P} 2$ & 0.92 & 32.48 & 55.34 & 11.26 & 0.00 \\
\hline & P3 & 1.40 & 32.03 & 51.65 & 14.79 & 0.13 \\
\hline & $\mathrm{P} 4$ & 1.05 & 31.67 & 53.33 & 13.95 & 0.00 \\
\hline & P5 & 0.98 & 40.49 & 54.06 & 4.47 & 0.00 \\
\hline & P6 & 1.02 & 37.71 & 54.13 & 7.14 & 0.00 \\
\hline & P7 & 1.09 & 38.61 & 53.99 & 6.32 & 0.00 \\
\hline & Average & 1.08 & 36.02 & 53.81 & 9.08 & 0.02 \\
\hline \multirow[t]{8}{*}{ Shield dune } & P1 & 1.51 & 39.87 & 52.36 & 6.07 & 0.19 \\
\hline & P2 & 1.29 & 35.06 & 52.70 & 10.96 & 0.00 \\
\hline & P3 & 1.67 & 30.96 & 49.02 & 17.74 & 0.60 \\
\hline & P4 & 1.01 & 32.87 & 53.79 & 12.25 & 0.09 \\
\hline & P5 & 1.24 & 43.51 & 51.82 & 3.44 & 0.00 \\
\hline & P6 & 0.95 & 39.58 & 54.14 & 5.25 & 0.08 \\
\hline & P7 & 1.34 & 37.92 & 52.35 & 8.20 & 0.19 \\
\hline & Average & 1.29 & 37.11 & 52.31 & 9.13 & 0.16 \\
\hline \multirow{10}{*}{$\begin{array}{l}\text { Incipient crescent } \\
\text { dune }\end{array}$} & P1 & 1.21 & 44.79 & 51.60 & 2.39 & 0.00 \\
\hline & $\mathrm{P} 2$ & 1.17 & 38.86 & 53.48 & 6.48 & 0.00 \\
\hline & $\mathrm{P} 3$ & 1.33 & 41.59 & 53.00 & 4.08 & 0.00 \\
\hline & $\mathrm{P} 4$ & 1.26 & 38.95 & 52.97 & 6.81 & 0.02 \\
\hline & P5 & 1.33 & 43.68 & 51.64 & 3.26 & 0.09 \\
\hline & P6 & 0.71 & 31.77 & 55.89 & 11.55 & 0.08 \\
\hline & P7 & 0.76 & 31.17 & 55.82 & 12.26 & 0.00 \\
\hline & P8 & 0.99 & 39.25 & 54.25 & 5.51 & 0.00 \\
\hline & P9 & 0.91 & 41.11 & 54.03 & 3.95 & 0.00 \\
\hline & Average & 1.08 & 39.02 & 53.63 & 6.25 & 0.02 \\
\hline \multirow[t]{11}{*}{ Mature crescent dune } & P1 & 1.41 & 41.76 & 52.64 & 4.20 & 0.00 \\
\hline & $\mathrm{P} 2$ & 1.44 & 41.23 & 51.78 & 5.47 & 0.09 \\
\hline & P3 & 1.80 & 44.11 & 49.83 & 4.05 & 0.21 \\
\hline & $\mathrm{P} 4$ & 1.22 & 39.70 & 52.91 & 6.09 & 0.08 \\
\hline & P5 & 1.21 & 39.38 & 53.33 & 6.09 & 0.00 \\
\hline & P6 & 0.84 & 33.34 & 56.31 & 9.32 & 0.19 \\
\hline & P7 & 0.46 & 29.73 & 58.38 & 11.43 & 0.00 \\
\hline & P8 & 0.96 & 40.83 & 53.93 & 4.28 & 0.00 \\
\hline & P9 & 0.53 & 28.75 & 56.30 & 13.85 & 0.57 \\
\hline & $\mathrm{P} 10$ & 0.97 & 39.13 & 53.34 & 6.42 & 0.14 \\
\hline & Average & 1.08 & 37.80 & 53.87 & 7.12 & 0.13 \\
\hline
\end{tabular}

The grain size distribution for the different developmental stages showed strongly similar unimodal curves (Fig. 3). The grain size distribution was dominated by particles with diameters between 1.5 and $4.5 \varphi$. The particle sizes from 2.65 to $3.65 \varphi$ were slightly more abundant in incipient and mature crescent dunes. In contrast, particle sizes from 0.16 to $2.65 \varphi$ were slightly more abundant in oval and shield dunes. The peak values in the distribution were $2.82 \varphi$ for oval sand piles and $2.99 \varphi$ for the other three stages. However, the surface sediments did not differ greatly among the developmental stages. 


\subsection{Grain size parameter characteristics}

\subsubsection{Mean grain size}

The mean grain size for the four dune developmental stages ranged between 2.8 and $3.1 \varphi$, with a mean of $2.97 \varphi$ (Fig. 4a), similar to the mean grain size $(3.05 \varphi)$ found at many locations in the Taklimakan Desert (Ji, 1996). From oval sand piles to incipient crescent dunes, the range of diameters in the grain size distribution gradually increased (Table 3). During the evolution of the crescent dunes, mean grain size was smallest for incipient crescent dunes $(3.00 \varphi)$, followed by mature crescent dunes $(2.99 \varphi)$ and was largest for oval sand piles $(2.94 \varphi)$. The coefficient of variation for grain size in mature crescent dunes (0.043) was higher than those in the other three developmental stages (ca. 0.030), but the differences were not significant (Table 3).

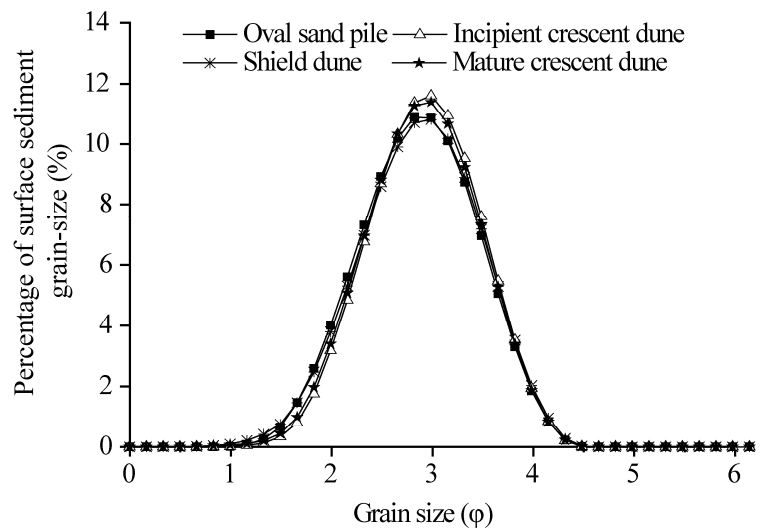

Fig. 3 Surface sediment grain size distribution for the four developmental stages of crescent dune

Table 3 Characteristics of the grain size distribution for crescent sand dunes at four developmental stages

\begin{tabular}{ccccccc}
\hline Development stage & Grain size range $(\varphi)$ & Average $(\varphi)$ & $\begin{array}{c}\text { Coefficient } \\
\text { of variation }\end{array}$ & $\begin{array}{c}\text { Maximum } \\
\text { point }\end{array}$ & $\begin{array}{c}\text { Minimum } \\
\text { point }\end{array}$ & Range $(\varphi)$ \\
\hline Oval sand pile & $2.85-3.03$ & $2.94 \pm 0.09^{\mathrm{a}}$ & $0.030^{\mathrm{a}}$ & $\mathrm{P} 3$ & P5 & 0.18 \\
Shield dune & $2.84-3.04$ & $2.96 \pm 0.09^{\mathrm{a}}$ & $0.030^{\mathrm{a}}$ & $\mathrm{P} 3$ & $\mathrm{P} 5$ & 0.19 \\
Incipient crescent dune & $2.86-3.08$ & $3.00 \pm 0.09^{\mathrm{a}}$ & $0.030^{\mathrm{a}}$ & $\mathrm{P} 7$ & $\mathrm{P} 5$ & 0.22 \\
Mature crescent dune & $2.80-3.09$ & $2.99 \pm 0.13^{\mathrm{a}}$ & $0.043^{\mathrm{a}}$ & $\mathrm{P} 9$ & $\mathrm{P} 10$ & 0.28 \\
\hline
\end{tabular}

Note: Different lowercase letters indicate significant difference at $P<0.05$ level. The locations of the maximum and minimum points are shown in Fig. 2.

\subsubsection{Sorting}

Sorting reflects the complexity of near-surface dynamic aeolian processes, and sorting is better when aeolian dynamics are stable (Shanbei Department, Chengdu Institute of Geology, 1978). In contrast, the more complex the aeolian dynamics, the poorer the sorting. The sorting values of 0.5 to 0.7 for the four crescent dune developmental stages represented that moderately well sorted the sediments was (Fig. 4b). Sorting of particles in the incipient and mature crescent dunes was similar (0.54) and showed relatively little variation among positions, and these sediments were slightly better sorted than those in oval sand piles (0.57) and shield dunes (0.58), which showed more variation. This showed that during the evolution of crescent dunes, sediments generally became better sorted as the dunes matured.

\subsubsection{Kurtosis and skewness}

Kurtosis values of the surface sediments (Fig. 4c) ranged from 0.465 to 0.841 , with an average of 0.579 , which represents platykurtic to very platykurtic kurtosis. The values followed a similar trend to the sorting values were discussed in the previous section. Shield dunes had the highest kurtosis values and the greatest variation among different positions within the dune, followed by 
oval sand piles and mature crescent dunes; incipient crescent dunes had the lowest values. During the evolution from oval to incipient crescent dunes, sediments generally tended to exhibit wider kurtosis; that is, the sediments became less variable in their composition.

Skewness is a quantitative indicator of the symmetry of the grain size distribution, and therefore provides an indication of whether the distribution is biased towards a given size class (Shanbei Department, Chengdu Institute of Geology, 1978). The skewness values for the surface sediments ranged from -0.126 to 0.003 (Fig. 4d), and averaged -0.025 ; this overall negative skewness indicated that the grain size of the sediments was biased towards coarser materials. The skewness value of shield dunes was lowest (a mean of -0.057), intermediate and similar for the oval sand piles and mature crescent dunes (both averaging -0.021), and highest for incipient crescent dunes $(-0.014)$.

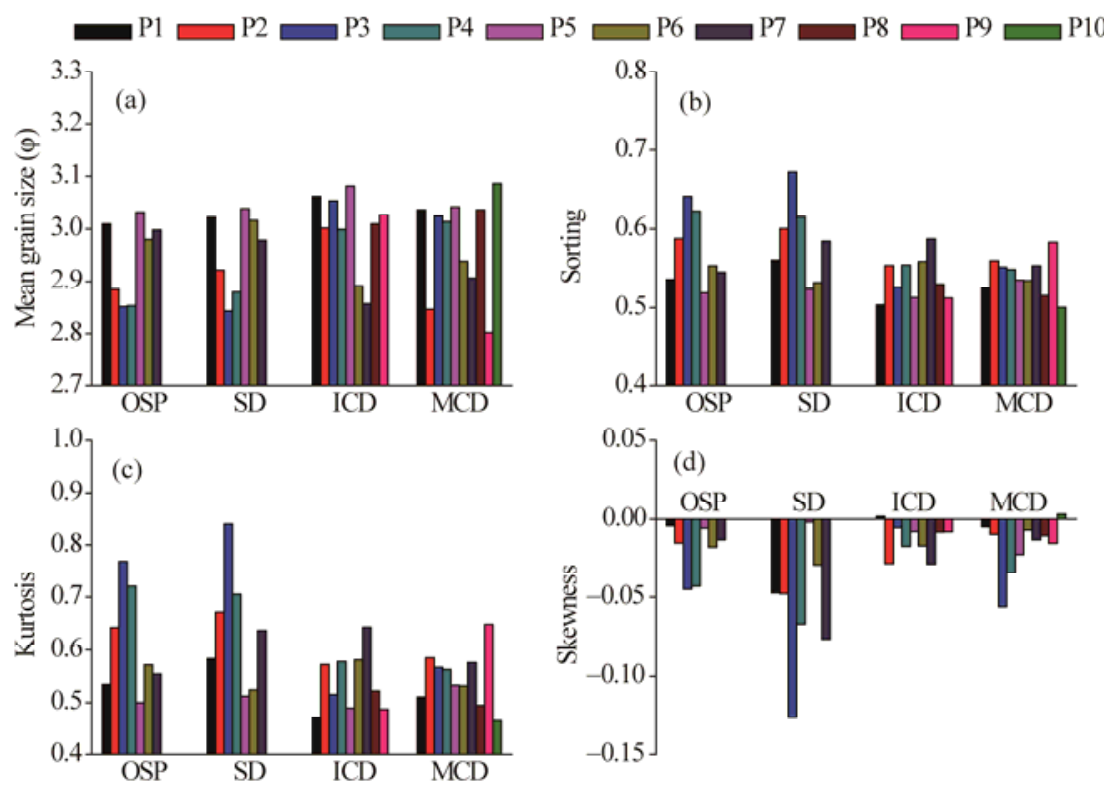

Fig. 4 Parameters of the surface sediment grain size distributions for the four different stages of dune evolution. OSP, oval sand piles; SD, shield dunes; ICD, incipient crescent dunes; MCD, mature crescent dunes.

\subsubsection{Correlations between the grain size parameters}

Correlations between the mean grain size and other grain size parameters can be used to reveal the characteristics of the sedimentary environment. Figure 5 presents scatterplots of the sorting, skewness and kurtosis as a linear function of the mean grain size for the four developmental stages. With mean grain sizes getting finer, sorting generally became better (Fig. 5a), skewness values did not show obvious changing trend (Fig. 5b) and kurtosis became wider (Fig. 5c). These results are similar to previously reported trends for a range of sand dune types (Lancaster, 1995). However, we found some differences in the correlations among the developmental stages. For example, oval sand piles and mature crescent dunes showed a symmetrical skewness that was relatively independent of mean grain size, whereas shield dunes showed a wide range of skewness values for a given mean grain size. From oval sand piles to shield and incipient crescent dunes to mature crescent dunes, the slopes of the linear regression curves between mean grain size and sorting decreased in order, as well as the slopes of the linear regression curves between mean grain size and kurtosis.

\subsection{Effects of aeolian activity on grain size distribution}

We compared the wind velocity, sand transport rates and wind erosion depths among the crescent dune developmental stages (Fig. 6). The wind velocities at a height of $2 \mathrm{~m}$ and sand transport rates in the $20-\mathrm{cm}$ layer above the surface did not differ greatly among the stages of dune 

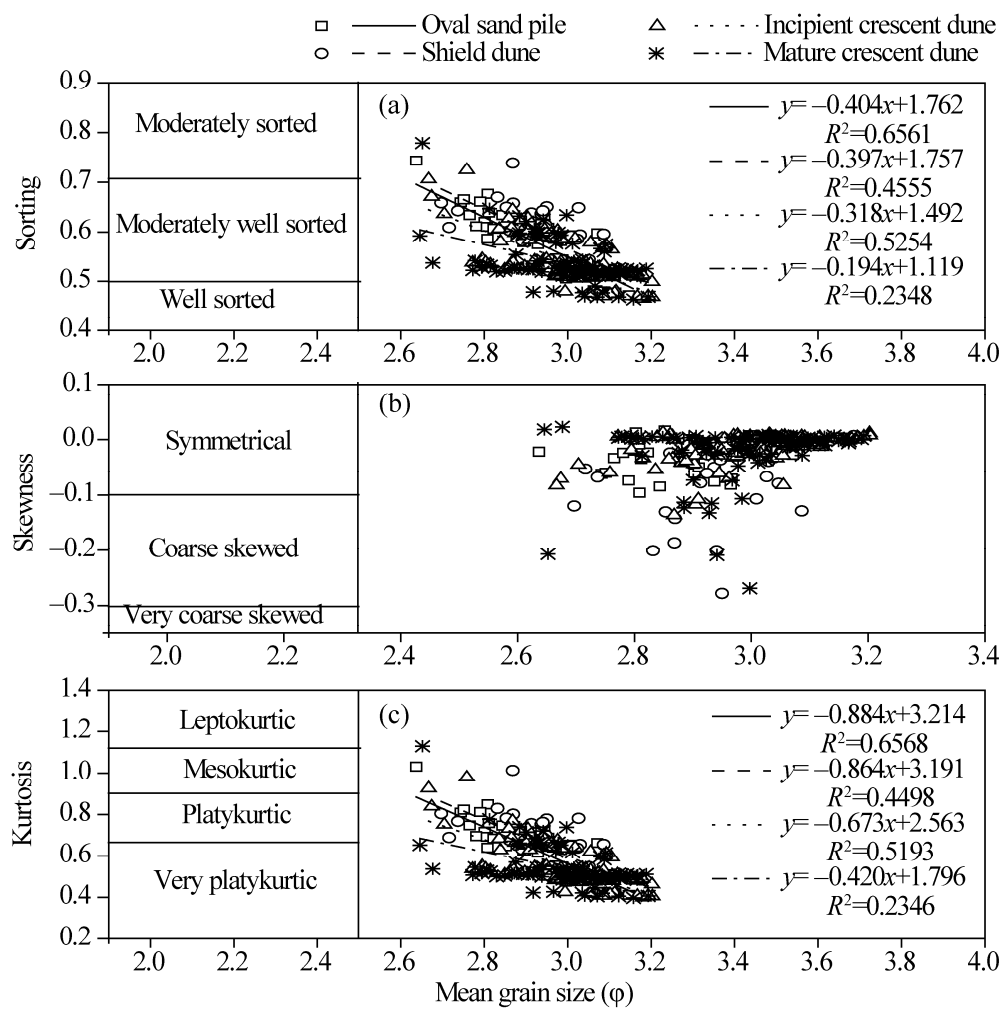

Fig. 5 Correlations between mean grain size and the sorting (a), skewness (b) and kurtosis (c) of the surface sediments at the four dune developmental stages

evolution. Both wind velocities and sand transport rates gradually increased moving from the toe of the windward slope to the top of the dune, and then gradually dropped from the top to the bottom of the leeward slope. Wind velocities and sand transport rates were highest at the top of the dunes. For the wind erosion/deposition depth, we found the strongest erosion at the middle of the windward slope and weak erosion at the top of the dunes. Aeolian deposition occurred on the leeward slope, with the greatest deposition occurring at the foot of the leeward slope.

Table 4 provides the slope angle at different locations on the windward slope of the dunes; we calculated these values based on the topographic survey data. The slope angle on oval and shield dunes gradually decreased moving from the toe of the windward slope to the top of the dune, and the windward slope was convex. For the incipient and mature crescent dunes, slope angles increased from the toe of the windward slope to the lower middle section, decreased at the middle of the windward slope, increased at the upper middle of the windward slope, and then decreased again at the top of the dune. Dune morphology determines the shape of the near-surface airflow. Sand flow also plays an important role in the formation and evolution of that surface. The interaction between dune morphology and sand flow determines the grain size distribution (Wang et al., 2002; Schatz and Herrmann, 2006).

The mean grain size was coarsest for the oval sand piles, followed by the shield dunes and mature crescent dunes, and was finest for the incipient crescent dunes. This is likely to be related to the rate at which the dunes migrated. During the observation period ( $30 \mathrm{~d})$, oval sand piles migrated most rapidly with an average migration distance of $1.28 \mathrm{~m}$, followed by shield dunes $(1.09 \mathrm{~m})$ and mature crescent dunes $(0.99 \mathrm{~m})$. The incipient crescent dunes migrated slowest $(0.92$ $\mathrm{m})$. This indicated that the faster the dune migrated, the coarser the grain size in the study area. This is because aeolian environment is characterized by comparatively strong wind and unsaturated sand flow in the open ground among tall-complex longitudinal ridges, which is unfavorable for the accumulation of finer sand particles but favorable for dunes migration. 
Similarly, the mean grain size of the surface sediments on the left (ESE) sides of the dunes was generally finer than on the right (WNW) sides during all four developmental stages, thus the right (WNW) wings moved faster than the left (ESE) wings in the prevailing wind direction.
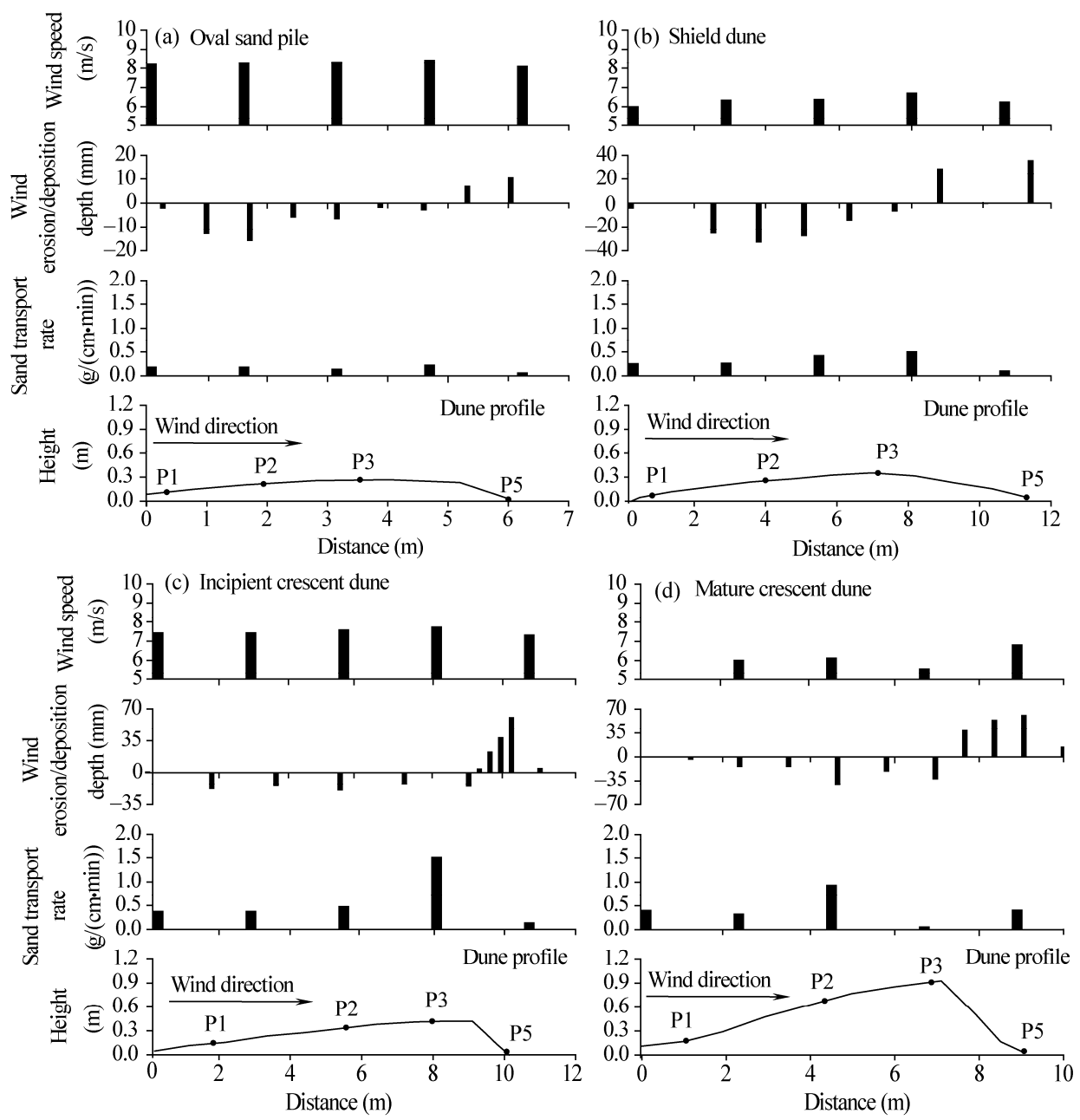

Fig. 6 Characteristics of the wind erosion and transport regimes during the four crescent dune developmental stages. Wind velocity was measured at a 2-m height above the surface, and the sand transport rate represents the total rate from the surface to $20 \mathrm{~cm}$. The wind erosion/deposition depth represents the total change in elevation of the surface over a period of 1 month; negative values represent erosion and positive values represent deposition.

Table 4 Angle of the windward slope at different positions on the dune

\begin{tabular}{lccccc}
\hline & \multicolumn{5}{c}{ Slope angle $\left(^{\circ}\right)$} \\
\cline { 2 - 6 } Development stage & $\begin{array}{c}\text { Toe of the } \\
\text { windward slope }\end{array}$ & $\begin{array}{c}\text { Lower middle } \\
\text { section of the } \\
\text { windward slope }\end{array}$ & $\begin{array}{c}\text { Middle section of } \\
\text { the windward slope }\end{array}$ & $\begin{array}{c}\text { Upper middle } \\
\text { section of the } \\
\text { windward slope }\end{array}$ & $\begin{array}{c}\text { Top of the } \\
\text { dune }\end{array}$ \\
\hline $\begin{array}{l}\text { Oval sand pile } \\
\text { Shield dune }\end{array}$ & 16.12 & 12.90 & 9.17 & 1.79 & 0.00 \\
$\begin{array}{l}\text { Incipient crescent } \\
\text { dune }\end{array}$ & 15.47 & 9.85 & 6.75 & 4.46 & 0.00 \\
$\begin{array}{l}\text { Mature crescent } \\
\text { dune }\end{array}$ & 6.87 & 12.36 & 6.95 & 9.64 & 0.45 \\
\hline
\end{tabular}




\section{Discussion}

Our results suggest that the grain size distribution of surface sediments in oval and shield dunes were similar. Dune development stages both had the coarsest grains at the top of the dunes, with finer grains at the toe of the windward slope, the bottom of the leeward slope, and both sides of the dunes. The mean grain sizes at the top of the two dune types were 2.85 and $2.84 \varphi$, respectively. Mean grain sizes were finest at the bottom of the leeward slope (3.04 and $3.08 \varphi$, respectively). The grain size distribution of the surface sediments of incipient and mature crescent dunes was also similar. For these two dune stages, mean grain size was coarser in the middle of the windward slope and at the top of the dune's trailing crest lines than at other dune positions. Moreover, mean grain size was coarser at the two sides of dunes in all four developmental stages and the two wings in the incipient and mature crescent dunes. The characteristics of the grain size distribution in our study differed somewhat from those in previous studies of crescent dunes by Vincent (1984) in the An Nafud Desert, by Watson (1986) in the Namib and Al Jafurah deserts, and by Lancaster (1995) in the Namib Desert.

The grain size distribution evolves in response to windblown sand activity. In addition, when air flows over the oval and shield dunes, the flow will separate on the leeward slope, thereby forming vortices with a horizontal axis of rotation before slowing down and depositing some of the sand it carries (Wu, 2003; Schatz and Herrmann, 2006; Ding, 2010). This causes airborne sand to continuously accumulate below the vortex that forms above the leeward slope, although finer particles are transported farther down the leeward slope to accumulate. The slip face had not yet formed during these two stages in dune development. The shield dunes were taller and their leeward slopes were longer than those of oval sand piles; thus, the degree of attenuation of airflow on the leeward slope was greater than on oval sand piles, the grain size of the leeward slope on the shield dunes was generally finer than on oval sand piles, and the distribution range of grain sizes was broader than on oval sand piles.

Changes for the angles of the windward slope of the incipient and mature crescent dunes were complex (Table 4), resulting in continuous changes in near-surface airflow. At the bottom of the windward slope, the slope angle was smaller and increased near the top of the dune. It then decreased in the middle of the windward slope before increasing again, creating a concave shape in the middle section. This phenomenon caused near-surface wind flow to converge and increase, which would lead to more intense erosion; as a result, primarily the coarsest grains remained at this position. The slope angle then decreased, creating a convex shape near the top of the dune. Although wind velocity was largest at this position, a convex slope generally decreased the near-surface wind velocity (Zhang et al., 1999) and weakened erosion processes, leading to deposition. Thus, the grain size at this position was finer than that in the middle of the windward slope.

The leeward slope of the incipient and mature crescent dunes is formed by sand on the slip face. Sand accumulated at the bottom of the leeward slope by sliding downhill under the influence of gravity. Vortices that formed on the leeward side of the dunes can pull finer sand upwards from the bottom to the middle of the leeward slope (Anderson and Haff, 1988; Liu et al., 1999; Tsoar, 2006; Livingstone, 2013). Therefore, the grain size in the middle of the leeward slope was finer than that at the bottom of the slope. Hence, due to the existence of a slip face, the grain size distribution in incipient and mature crescent dunes differed from those in the two earlier stages of dune development.

As a transitional stage between shield and mature crescent dunes, incipient crescent dunes exhibited a gradual windward slope similar to that of shield dunes but exhibited a slip face similar to that of mature crescent dunes. Thus, sediment characteristics related to grain size are also intermediate between the early and mature stages of dune development. Compared to the other three stages, mature crescent dunes were taller and exhibited a typical slip face. They also had a strong impact on airflow, resulting in intense wind erosion on the windward slope and continuous deposition on the leeward slope. Differences in the grain size distribution largely resulted from the positions of erosion and deposition on the sand dune and led to the largest distribution range 
in grain size (Table 1).

Although sorting and kurtosis values were negatively correlated with mean grain size $(\varphi)$ in all four dune developmental stages, the significance of the correlation differed. As mean grain size $(\varphi)$ increased, the sorting and kurtosis values decreased more rapidly for oval and shield dunes than for incipient and mature crescent dunes. This was due to the lower heights of the oval and shield dunes and the weaker flow-separation effects for near-surface airflow on the leeward sides of these dunes, which resulted in the differentiation of grain size parameters from those of the two most mature stages. Therefore, the differences in dune morphology created variations in dynamic conditions and dynamic processes, which in turn created variations in the grain size distribution among stages.

\section{Conclusions}

The crescent dunes of the four developmental stages in the hinterland of the Taklimakan Desert were dominated by fine sands, followed by very fine and medium sands. Coarse silts and coarse sands accounted for very small proportions of the total, and no particles finer than coarse silt or coarser than coarse sand. The grain size distribution curves all showed similar normal distributions. The surface sediments were generally moderately well sorted. Kurtosis was generally platykurtic or very platykurtic, and the distribution was skewness towards the coarser particles.

We found similar correlations between mean grain size and the other sediment parameters for all four dune developmental stages. During the evolution towards mature crescent dunes, mean grain size $(\varphi)$ increased; sorting improved, kurtosis widened and skewness did not change greatly. However, there were some differences in the grain size distribution among the four stages. For the oval and shield dunes, particles at the top were the coarsest; for the incipient and mature crescent dunes, particles at the middle and upper middle parts of the windward slopes were the coarsest and were finer at their tops. These similarities and differences among the stages resulted from interactions between dune morphology and the surface airflow, leading to variation in the intensities of erosion and deposition at different positions on the dunes.

Because we did not obtain enough sand transport data to model the transport flux as a function of a height, and did not measure the wind velocity fields above the windward and leeward slopes of the dunes, we cannot fully confirm some of our proposed explanations of the results. In future research, it will be necessary to obtain this data and determine its implications for the formation and evolution of crescent dunes.

\section{Acknowledgements}

This research was supported by the National Natural Science Foundation of China $(41571011,41330746)$.

\section{References}

Anderson R S, Haff P K. 1988. Simulation of Eolian saltation. Science, 241(4867): 820-823.

Bagnold R A. 1941. The Physics of Blown Sand and Desert Dune. London: Methuen, 85-96.

Ding G D. 2010. Aeolian Physics (2 ${ }^{\text {nd }}$ ed.). Beijing: China Forestry Publishing House, 105-110. (in Chinese)

Folk R L, Ward W C. 1957. Brazos river bar: a study in the significance of grain size parameters. Journal of Sedimentary Petrology, 27(1): 3-26.

Ha S, Wang G Y, Dong G R. 2001. Lee-face airflow, depositional types and its significance. Acta Sedimentologica Sinica, 19(1): 96-100. (in Chinese)

Hesp P A, Hastings K. 1998. Width, height and slope relationships and aerodynamic maintenance of barchans. Geomorphology, 22(2): 193-204.

Ji Q H. 1996. Application of grain size analysis in the studies of Taklimakan Desert. Journal of Desert Research, 16(2): 173-179. (in Chinese)

Lancaster N. 1985. Variations in wind velocity and sand transport on the windward flanks of desert sand dunes. Sedimentology, 32(4): 581-593. 
Lancaster N. 1989. The Namib Sand Sea: Dune Forms, Processes and Sediments. Rotterdam: Balkema, 192.

Lancaster N. 1995. Geomorphology of Desert Dunes. New York: Routledge.

Lancaster N, Nickling W G, Neuman C M. 2002. Particle size and sorting characteristics of sand in transport on the stoss slope of a small reversing dune. Geomorphology, 43(3-4): 233-242.

Ling Y Q, Wu Z, Liu S Z, et al. 1998. Simulated study on barchan dune forms. Scientia Geographica Sinica, 18(1): 88-93. (in Chinese)

Liu X W, Li S, Shen J Y. 1999. Wind tunnel simulation experiment of mountain dunes. Journal of Arid Environments, 42(1): 49-59.

Livingstone I. 1989. Temporal trends in grain-size measures on a linear sand dune. Sedimentology, 36(6): 1017-1022.

Livingstone I. 2013. Aeolian geomorphology of the Namib Sand Sea. Journal of Arid Environments, 93: 30-39.

Nickling W G, Neuman C M, Lancaster N. 2002. Grainfall processes in the lee of transverse dunes, Silver Peak, Nevada. Sedimentology, 49(1): 191-209.

Pye K, Tsoar H. 1990. Aeolian Sand and Sand Dunes. London: Unwin Hyman, 396.

Schatz V, Herrmann H J. 2006. Flow separation in the lee side of transverse dunes: a numerical investigation. Geomorphology, 81(1-2): 207-216.

Shanbei Department, Chengdu Institute of Geology. 1978. Grain Size Analysis of Sediments (Rocks) and Its Application. Beijing: Geology Press, 55-133. (in Chinese)

Tsoar H. 2006. Dynamic processes acting on a longitudinal (seif) sand dune. Sedimentology, 30(4): 567-578.

Vincent P J. 1984. Particle size variation over a transverse dune in the Nafud as Sirr, central Saudi Arabia. Journal of Arid Environments, 7(4): 329-336.

Wang X M, Dong Z B, Zhang J W, et al. 2002. Relations between morphology, air flow, sand flux and particle size on transverse dunes, Taklimakan Sand Sea, China. Earth Surface Processes and Landforms, 27(5): 515-526.

Wang X M, Dong Z B, Zhang J W, et al. 2003. Grain size characteristics of dune sands in the central Taklimakan Sand Sea. Sedimentary Geology, 161(1-2): 1-14.

Watson A. 1986. Grain-size variations on a longitudinal dune and a barchan dune. Sedimentary Geology, 46(1-2): 49-66.

White B R, Tsoar H. 1998. Slope effect on saltation over a climbing sand dune. Geomorphology, 22(2): 159-180.

Wu Z. 2003. Geomorphology of Wind-drift Sands and Their Controlled Engineering. Beijing: Science Press, 165-170. (in Chinese)

Zhang C L, Hao Q Z, Zou X Y, et al. 1999. Response of morphology and deposits to surface flow on windward slope of barchan dune. Journal of Desert Research, 19(4): 359-363. (in Chinese)

Zhu Z D, Chen Z P, Wu Z. 1981. Study on Aeolian landform in Taklimakan Desert. Beijing: Science Press. (in Chinese)

Zu R P, Zhang K C, Qu J J, et al. 2005. The intensity of sand-drift activities in Taklimakan Desert. Geographical Research, 24(5): 699-707. (in Chinese) 